\title{
Enhanced Molecular Spin-Photon Coupling at Superconducting Nanoconstrictions
}

\author{
Ignacio Gimeno, Wenzel Kersten, María C. Pallarés, Pablo Hermosilla, María José Martínez-Pérez, \\ Mark D. Jenkins, Andreas Angerer, Carlos Sánchez-Azqueta, David Zueco, Johannes Majer, \\ Anabel Lostao, and Fernando Luis*
}

Cite This: ACS Nano 2020, 14, 8707-8715

Read Online

ACCESS | Llll Metrics \& More | 回 Article Recommendations | st Supporting Information

ABSTRACT: We combine top-down and bottom-up nanolithography to optimize the coupling of small molecular spin ensembles to $1.4 \mathrm{GHz}$ on-chip superconducting resonators. Nanoscopic constrictions, fabricated with a focused ion beam at the central transmission line, locally concentrate the microwave magnetic field. Drops of free-radical molecules have been deposited from solution onto the circuits. For the smallest ones, the molecules were delivered at the relevant circuit areas by means of an atomic force microscope. The number of spins $N_{\text {eff }}$ effectively coupled to each device was accurately determined
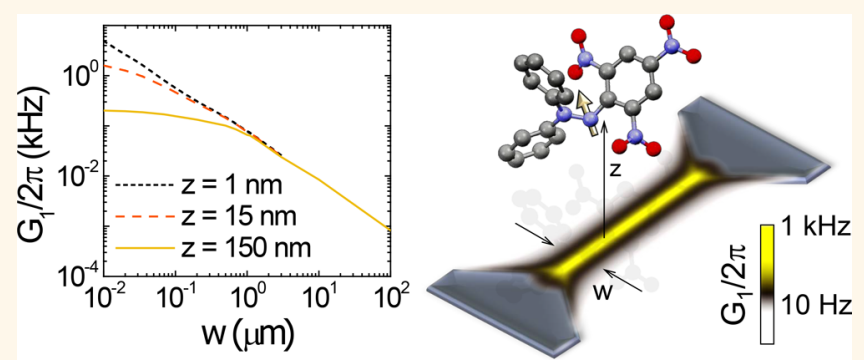
combining Scanning Electron and Atomic Force Microscopies. The collective spin-photon coupling constant has been determined for samples with $N_{\text {eff }}$ ranging between $2 \times 10^{6}$ and $10^{12}$ spins, and for temperatures down to $44 \mathrm{mK}$. The results show the well-known collective enhancement of the coupling proportional to the square root of $N_{\text {eff }}$ The average coupling of individual spins is enhanced by more than 4 orders of magnitude (from $4 \mathrm{mHz}$ up to above $180 \mathrm{~Hz}$ ), when the transmission line width is reduced from $400 \mu \mathrm{m}$ down to $42 \mathrm{~nm}$, and reaches maximum values near $1 \mathrm{kHz}$ for molecules located on the smallest nanoconstrictions.

KEYWORDS: molecular spins, superconducting resonators, electron spin resonance, dip pen nanolithography, focused ion beam nanolithography, spin qubits, circuit quantum electrodynamics

$\mathrm{T}$ he coupling of spins to superconducting circuits lies at the basis of diverse technologies. Superconducting onchip resonators, which concentrate the microwave magnetic field in much smaller regions than conventional three-dimensional cavities, ${ }^{1,2}$ promise to take electron spin resonance (ESR) to its utmost sensitivity level, eventually allowing the detection of single spins. ${ }^{3-6}$ Besides, single microwave photons "trapped" in these devices provide a way to wire-up qubits ${ }^{7-9}$ and, therefore, form a basis for hybrid quantum computation and simulation schemes based on spins. ${ }^{10-16}$

Different approaches have been designed and, in some cases, put into practice, to enhance the spin sensitivity and the spinphoton coupling. They often involve the use of nonlinear superconducting circuits, parametric amplifiers, to amplify the output signal, ${ }^{3,4}$ and of low impedance resonator designs, which increase the photon magnetic field. ${ }^{4,5}$ Experiments performed on highly coherent magnetic impurities in semiconducting hosts show spin resonance at the level of a few tens of spins and maximum spin-photon coupling strengths of order $400 \mathrm{~Hz}$ at frequencies of about $7 \mathrm{GHz} .^{5}$
In this work, we explore experimentally a third alternative, inspired by earlier developments of micro-ESR devices. ${ }^{17-19}$ The underlying idea is that reducing the cavity effective volume enhances the microwave magnetic field. In a superconducting resonator, this goal can be achieved by decreasing locally the width of the resonator's transmission line ${ }^{20-22}$ in order to bridge the very different scale lengths of superconducting circuits, with typical line widths of a few microns, and of impurity or molecular spins, which are in the range of nanometers. This approach requires moving beyond the limits of conventional optical lithography to fabricate or modify certain regions of the circuits and is fully complementary to those methods mentioned above.

Received: April 15, 2020

Accepted: May 22, 2020

Published: May 22, 2020 


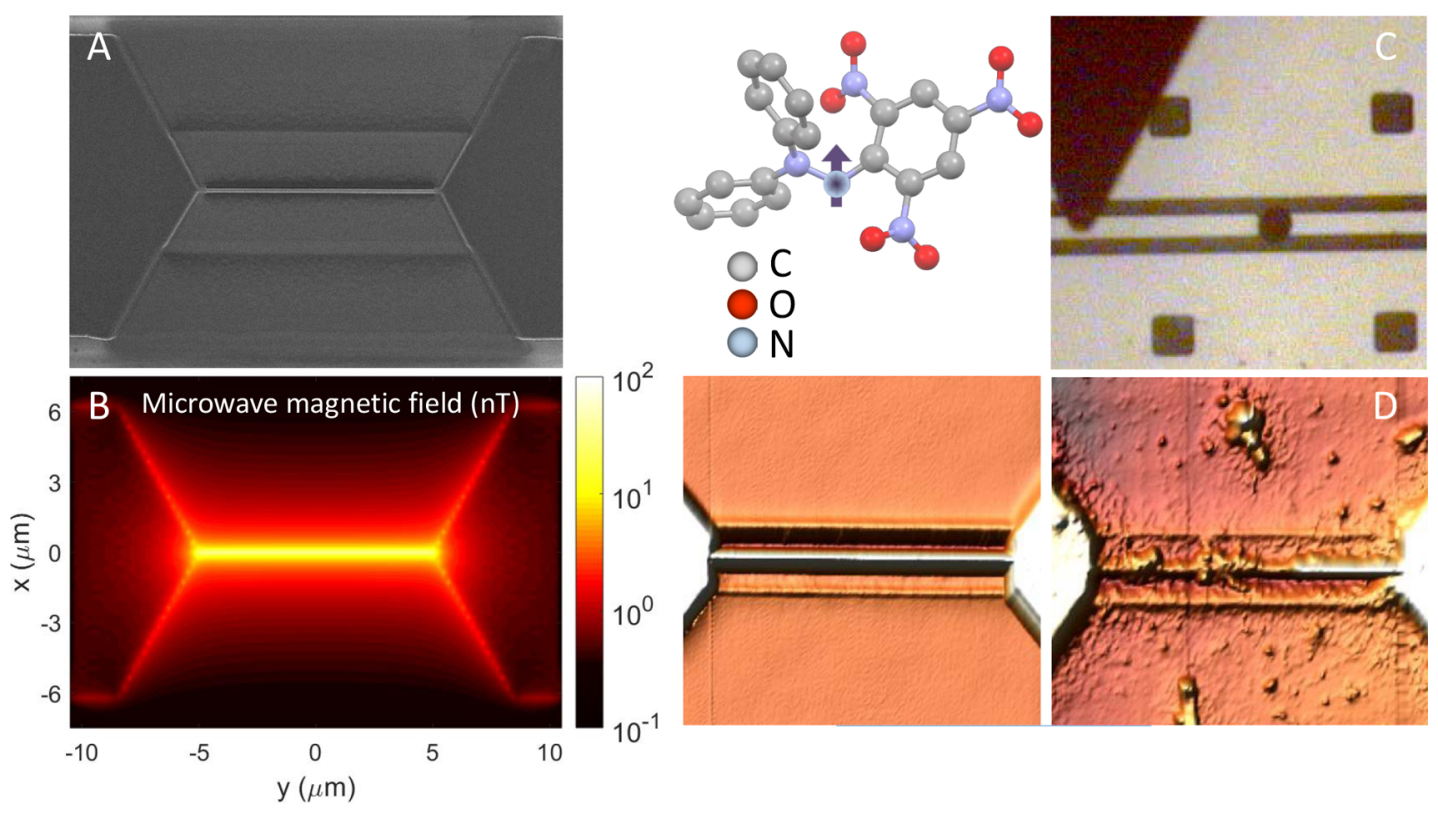

Figure 1. (A) Scanning electron microscopy image of the central line of a superconducting coplanar resonator. The line was thinned down to a width of about $158 \mathrm{~nm}$ by focused ion beam nanolithography. (B) Color plot of the photon magnetic field in the neighborhood of this constriction, calculated with a finite-element simulation software. ${ }^{35}$ (C) Structure of a DPPH free-radical molecule, with spin $S=1 / 2$ and $g=$ 2 (left) and optical microscopy image of the constriction after the deposition of DPPH by means of the tip of an Atomic Force Microscope (AFM, right). (D) AFM image of the constriction taken before and after the molecules were deposited and the solvent had evaporated.

The main challenge resides in the fact that the microwave field enhancement is localized in a nanoscopic region near the superconducting constriction, as can be seen in Figure 1A and 1B. Optimally profiting from such enhancement thus calls for a method able to deliver the magnetic sample at the right location and with sufficient spatial accuracy. Spins in molecules provide a good test case for addressing this challenge. Many molecules are stable in solution or can be sublimated from the crystal and can, therefore, be transferred to a solid substrate or a device. ${ }^{23-28}$ In addition, they are one of the most promising candidates to encode spin qubits, due to the vast possibilities for design offered by chemical nanoscience. ${ }^{29-34}$ Here, we combine top-down and bottom-up nanolithography methods to study the coupling of microwave photons to nanoensembles of the simplest molecular spins, organic free radicals with $S=$ $1 / 2$, deposited near constrictions of varying size.

\section{RESULTS AND DISCUSSION}

This section describes the main results of this work: how devices are modified to locally enhance the spin-photon coupling, how the molecules are deposited with sufficient spatial accuracy, and how this coupling is experimentally determined as a function of the number of molecules and of temperature. The main result refers to the coupling of individual spins and its dependence on the size of the resonator dimensions and on the molecule-to-chip interface.

Circuit Fabrication and Integration of Molecular Spin Micro- and Nano-deposits. The circuits used in this work are coplanar superconducting resonators fabricated by optical lithography on $150 \mathrm{~nm}$ thick $\mathrm{Nb}$ films, which become superconducting below $T_{\mathrm{c}}=8.2 \mathrm{~K}$, deposited onto single crystalline sapphire wafers. Previous studies ${ }^{21}$ show that nanoscopic constrictions, such as the one shown in Figure $1 \mathrm{~A}$, can be fabricated in the central transmission line by Focused Ion Beam (FIB) nanolithography, that they do not significantly alter the operation of the devices, and that they locally enhance the photon magnetic field.

As the simulation in Figure 1B shows, the enhancement is strongly localized near the superconducting nanobridge, in a region with typical dimensions $w \times w \times L$, where $w$ is the constriction width and $L$ is its length. The proper integration of spins plays, then, a crucial role to achieve the maximum spin sensitivity allowed by this approach. Our samples contain organic free-radical molecules of 2, 2-diphenyl-1-picrylhydra$\mathrm{zyl}^{36-38}$ hereafter referred to as DPPH, whose molecular structure is shown in Figure 1. Each molecule hosts an unpaired electron with a spin $S=1 / 2$ and a close to isotropic gyromagnetic factor $g \simeq 2$. Under a magnetic field $H$, it shows a well-defined resonance line at a frequency $\Omega_{S}=\mu_{0} g \mu_{\mathrm{B}} H / \hbar$. In a crystalline environment, the inhomogeneous broadening arising from dipolar interactions is reduced by direct exchange interactions between nearest radicals. ${ }^{39,40}$ The resonance line width becomes then dominated by the homogeneous broadening $1 / T_{2}$, where $T_{2} \simeq 80-120 \mathrm{~ns}$ is the spin coherence time. Besides, these molecules can be dissolved and remain stable in diverse organic solvents. ${ }^{37}$

The latter property allows delivering precise amounts of DPPH molecules onto the central transmission lines of the resonators. Large (a few $\mathrm{mm}$ wide, a few microns thick) molecular ensembles have been deposited either in powder form or from solution, using a micropipette, onto conventional resonators with $w=400$ and $14 \mu \mathrm{m}$. The number of molecules was varied by controlling the concentration of the original solution, keeping the volume constant. Illustrative Scanning Electron Microscopy (SEM) images of such deposits are shown as part of the Supporting Information. They show that DPPH has a tendency to form quasi-spherical nanoaggregates.

For smaller deposits on narrower lines, we employed Dip Pen Nanolithography (DPN) ${ }^{41,42}$ a soft lithographic technique that uses the tip of an Atomic Force Microscope (AFM) to deposit nanoscopic volumes from a solution containing the 
molecules of interest onto a very small area (cf. Figure 1C). This technique has the advantage of combining high spatial resolution with good control over the molecular dose transferred to the substrate without the need for chemical functionalization, thus making it well suited for placing diverse nanosamples onto solid state sensors. ${ }^{25,43,44}$ The size of the deposits was controlled in this case by the diameter of the drops transferred by the AFM tip to the substrate, which depend on the contact time between both, and by their concentration. The deposits are then characterized by SEM and by AFM, as shown in Figure 1D and in the Supporting Information. These deposits also form nanoaggregates after the solvent has evaporated, with sizes ranging from $50 \mathrm{~nm}$ to above $200 \mathrm{~nm}$ depending on the initial concentration of the radical at the ink solution and the size of the deposited drop. This also means that the number of molecules actually transferred into the "active" region of the constriction might vary even between depositions performed under nominally identical conditions and, therefore, needs to be determined for each case.

Spin-Photon Coupling versus Spin Number. The coupling of the spins to the cavity photons, with frequency $\omega_{\mathrm{r}} / 2 \pi \simeq 1.4 \mathrm{GHz}$, reduces the microwave transmission, shifts $\omega_{\mathrm{r}}$, and broadens the resonance. All these effects become maximum when $\Omega_{S} \simeq \omega_{\text {r }}$, i.e. when spins and photons are brought into resonance with each other by an external magnetic field $H$. The transmission provides information on the collective coupling $G_{N}$ of the spin ensemble to the resonator, where $N$ denotes here the number of free-radical spins, as well as on the spin $\gamma$ and photon $\kappa$ characteristic line widths.

Illustrative results of transmission measurements performed at $T=4.2 \mathrm{~K}$ on resonators without and with a $158 \mathrm{~nm}$ wide constriction are shown in Figure $2 \mathrm{~A}$ and $2 \mathrm{~B}$, respectively. In both cases, the sample was a DPPH drop with an approximate diameter of about $30 \mu \mathrm{m}$ deposited onto the central line by DPN. This amount corresponds approximately to the sensitivity limit for the former device. By contrast, under the same conditions, the resonator with the constriction gives a clearly visible absorption signal, thus providing direct evidence for the enhancement of $G_{N}$.

In order to obtain quantitative estimates of this enhancement, and estimate the average coupling to individual spins, experiments on samples with decreasing $N$ were performed. The number of molecules effectively coupling to the resonator magnetic field was estimated from the geometry and topography of the deposits, taking into account the width of the transmission line near the deposit. For conventional resonators with a $14 \mu \mathrm{m}$ wide central line, all spins located in a $30 \mu \mathrm{m}$ wide region around it are counted. For a nanoconstriction, the "active" area is taken as a $2 \mu \mathrm{m}$ wide rectangular area around it.

Experiments and simulations, described below and in the Supporting Information, confirm that the coupling of spins located outside this region lies below the sensitivity limits and can, therefore, be safely neglected. Another aspect that needs to be taken into account is that these measurements were performed at a finite temperature $T=4.2 \mathrm{~K}$. This lowers the population difference, or equivalently the spin polarization $\left\langle S_{z}\right\rangle_{T} / S=\tanh \hbar \Omega_{S} / k_{\mathrm{B}} T$, between the ground and excited levels of the DPPH spins by a factor $6.7 \times 10^{-3}$ with respect to zero temperature. As a result, the number of spins that contribute to the net absorption at the given temperature is also reduced from $N$ to $N_{\text {eff }}=N\left\langle S_{z}\right\rangle_{T} / S$.
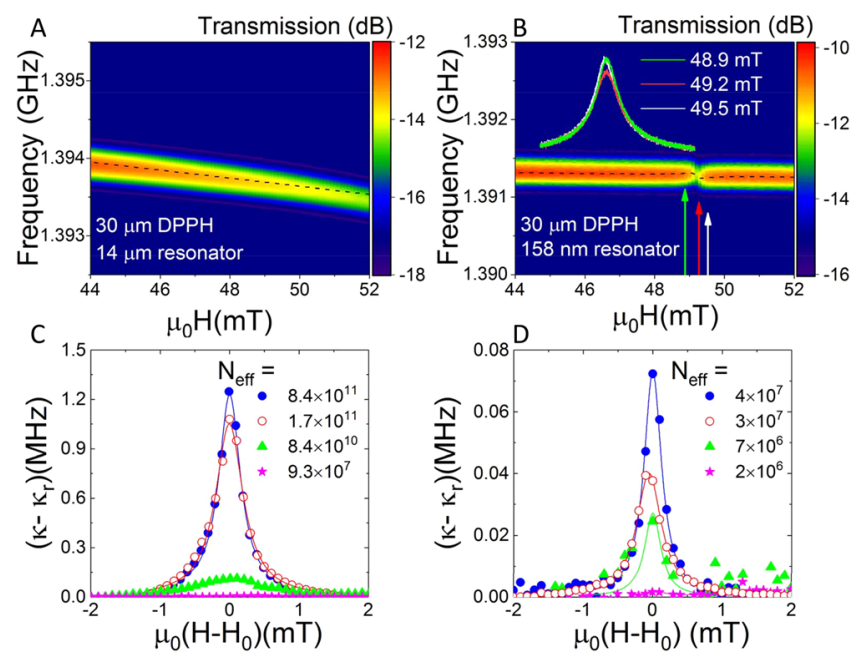

Figure 2. (Top) Color scale plots of the microwave transmission through $1.4 \mathrm{GHz}$ on-chip superconducting resonators with a 14 $\mu \mathrm{m}$ wide central line (A) and with a $158 \mathrm{~nm}$ wide constriction (B) coupled to a DPPH deposit with $N \approx 5 \times 10^{9}$ molecules, corresponding to $N_{\text {eff }} \approx 4 \times 10^{7}$ spins effectively coupled to the resonator at $T=4.2 \mathrm{~K}$. The red dashed lines mark the position of the resonance frequency at each magnetic field. The inset in $B$ shows transmission versus frequency data near resonance at the field values indicated by arrows, evidencing the detection of a net absorption (lower transmission and broader resonance) when the spins get into mutual resonance with the circuit. (Bottom) Magnetic field dependence of the resonance width $\kappa$ for the same resonators (C without and D with constriction) coupled to ensembles of DPPH molecules of varying size. Solid lines are leastsquares fits based on eq 1 .

Results from these experiments are shown in Figure 2C and 2D. They confirm that introducing a nanoconstriction enables detecting much smaller deposits: the sensitivity limit is reduced from about $10^{8}$ spins to $2 \times 10^{6}$ spins. This enhancement in sensitivity arises here from a larger spin-photon coupling, which can be determined as follows. The broadening of the cavity resonance $\kappa$ can be fitted using the following expression, $^{45}$

$$
\kappa=\kappa_{\mathrm{r}}+\frac{G_{N}^{2} \gamma}{\left(\omega_{\mathrm{r}}-\Omega_{S}\right)^{2}+\gamma^{2}}
$$

where $\kappa_{\mathrm{r}}$ is the broadening of the 'empty' cavity, as measured when it is detuned from the spins. This fit allows extracting $G_{N}$ and $\gamma . \gamma$ is found to be close to $12 \mathrm{MHz}$ for all but the smallest DPN deposits. This value is compatible with a pure homogeneous broadening and a $T_{2} \simeq 80 \mathrm{~ns}$, which lies within the typical values observed for DPPH. ${ }^{37} \mathrm{~A}$ larger line width has been observed in DPPH molecules dispersed in polymeric matrices. ${ }^{40}$ That the same phenomenon is observed in DPPH nanoaggregates deposited from solution suggests that they tend to lose crystalline order.

Figure 3 shows the dependence of $G_{N}$ on $N_{\text {eff }}$ measured in resonators with $14 \mu \mathrm{m}$ and $158 \mathrm{~nm}$ transmission line widths. In both, the coupling is approximately proportional to $N_{\text {eff }}^{1 / 2}$. This result agrees with the collective enhancement of the radiation emission and absorption by spin ensembles, ${ }^{46}$ which predicts that

$$
G_{N}=G_{1} N_{\text {eff }}^{1 / 2}
$$



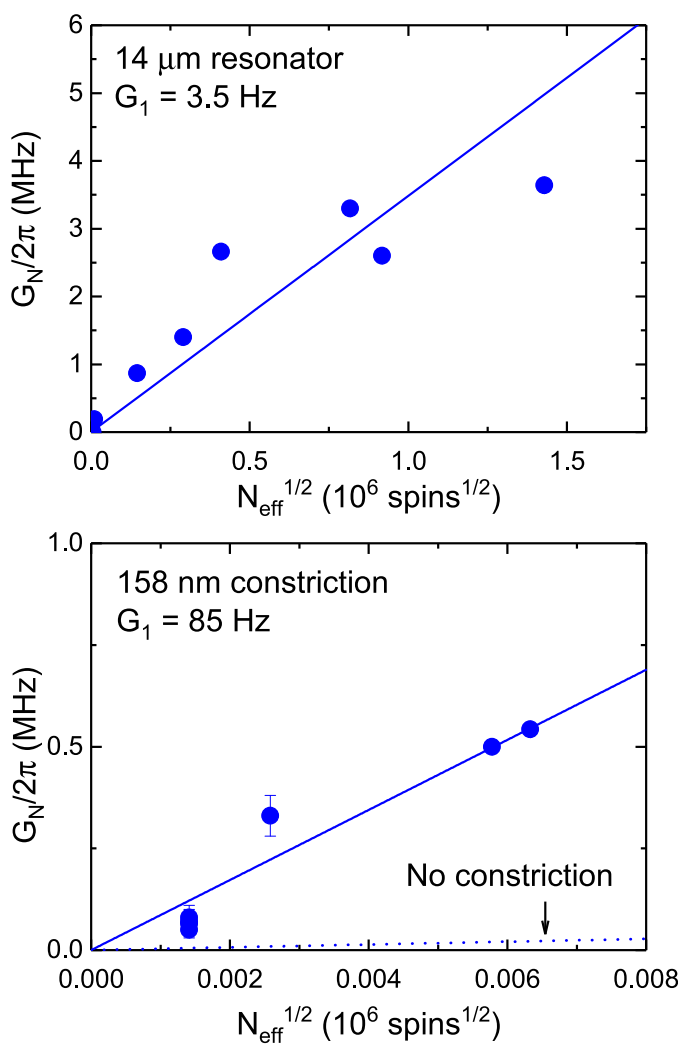

Figure 3. Collective spin-photon coupling of ensembles of freeradical molecules to coplanar resonators with a $14 \mu \mathrm{m}$ wide transmission line (top) and a $158 \mathrm{~nm}$ constriction (bottom). The solid lines are least-squares fits to a linear dependence on the square root of the effective number of spins that are coupled to the devices at $T=4.2 \mathrm{~K}$, as predicted by eq 2 . The bottom panel compares both fits to highlight the coupling enhancement generated by the constriction.

where $G_{1}$ is the coupling of a single spin. The ability to modify the size of the molecular ensembles over a large range allows monitoring this well-known dependence directly. The slope then directly gives the average, or typical, value of $G_{1}$. These data confirm also that a strong enhancement of $G_{1}$, by a factor of order 24 (from $G_{1} / 2 \pi \simeq 3.5$ to $85 \mathrm{~Hz}$ ), occurs when $w$ is reduced by a factor 100 (from $14 \mu \mathrm{m}$ to $158 \mathrm{~nm}$ ).

Spin-Photon Coupling versus Temperature. In this section, we describe experiments that explore the optimal conditions in the search of a maximum spin-photon coupling: temperatures close to absolute zero, which take $N_{\text {eff }}$ closer to $N$; an average number $n_{\text {photons }} \simeq 5 \times 10^{5}$ of photons in the cavity well below $N$, in order to avoid any saturation effects; and a central line width $w \simeq 42 \mathrm{~nm}$ near the minimum achievable by FIB nanolithography.

Representative images of the constriction and of the DPPH nanoaggregates deposited on it by DPN are shown in Figure 4 and in the Supporting Information. The number $N$ of DPPH molecules in the active area was, in this case, estimated to be approximately $1.6 \times 10^{8}$ spins, which lies below the minimum dose that was detectable in experiments performed at $T=4.2$ $\mathrm{K}$ and for $w=158 \mathrm{~nm}$ (cf. Figure 3D).

Results of transmission measurements performed on this device are shown in Figure 5. Thanks to the much larger spin polarization, an easily discernible absorption signal is observed at the minimum temperature $T \simeq 44 \mathrm{mK}$, which corresponds to a collective coupling $G_{N} / 2 \pi \simeq 1.9 \mathrm{MHz}$. The line width $\gamma \simeq$
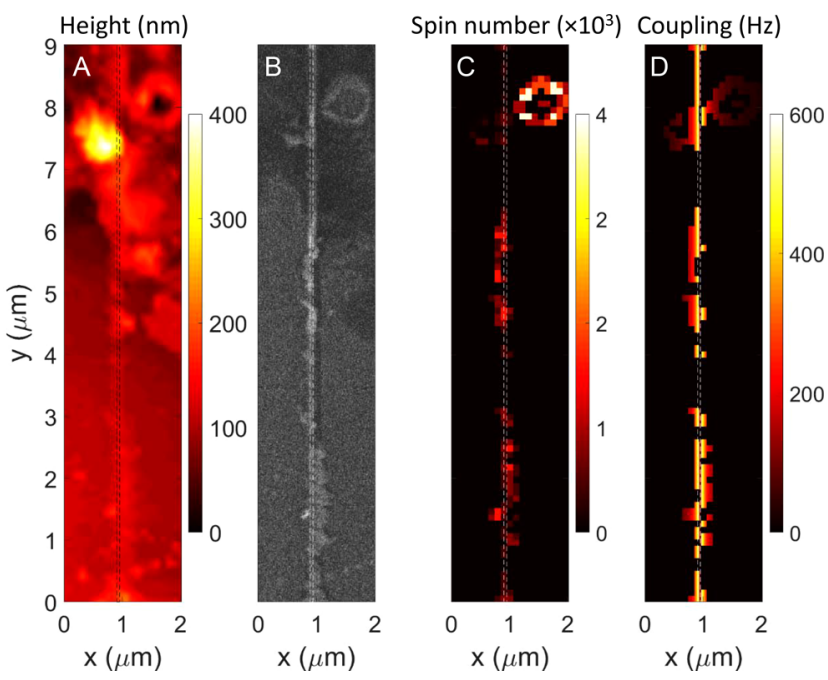

Figure 4. AFM topographic map (A) and SEM image (B) of the region near a $42 \mathrm{~nm}$ wide nanoconstriction. ( $C$ and $D$ ) Color maps of the number of DPPH molecules deposited on each location and of the estimated single spin to photon couplings, respectively. The latter maps have been calculated with a discretization of space into $3 \times 3 \times 3 \mathrm{~nm}^{3}$ cubic cells. The number of free-radical molecules deposited in this area amounts to approximately $N=1.6 \times 10^{8}$, and the collective coupling estimated from the simulations is $G_{N} /$ $2 \pi \simeq 2.0 \mathrm{MHz}$ at $T=44 \mathrm{mK}$ and $2.5 \mathrm{MHz}$ at $T=0$.
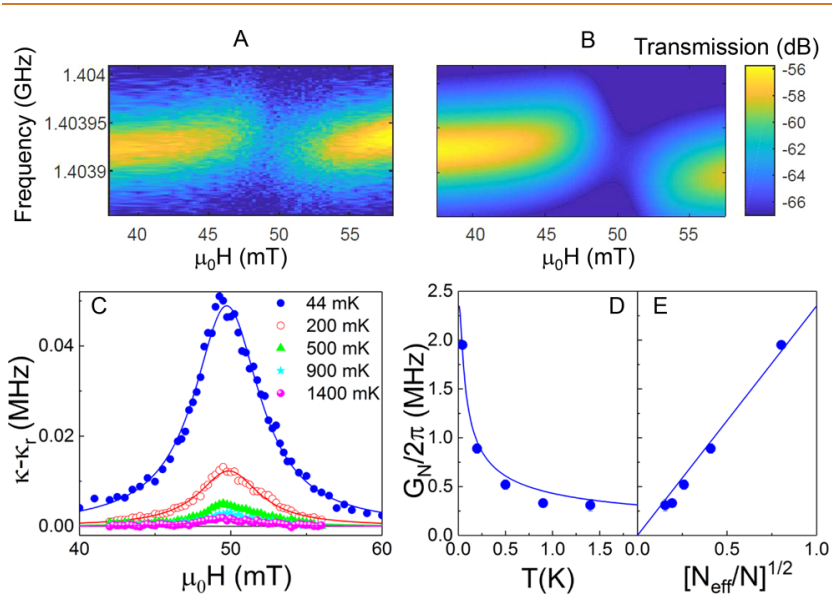

Figure 5. (A) Color plot of the microwave transmission, measured at $T=44 \mathrm{mK}$, through a superconducting resonator with a $42 \mathrm{~nm}$ wide constriction in its central transmission line and coupled to an ensemble of $N \simeq 1.6 \times 10^{8}$ free-radical molecules (corresponding to $N_{\text {eff }} \simeq 10^{8}$ spins effectively coupled to the resonator). (B) Color plot of the microwave transmission calculated for a collective coupling $G_{N} / 2 \pi=2.0 \mathrm{MHz}$, as follows from the simulations described in Figure 4, and a spin line width $\gamma=65 \mathrm{MHz}$. (C) Magnetic field dependence of the resonance width $\kappa$ for the same device measured at different temperatures. Solid lines are leastsquares fits using eq 2. (D) Temperature dependence of $G_{N}$ extracted from these experiments. (E) Same data plotted as a function of the (temperature dependent) effective number of spins coupled to the resonator $N_{\text {eff }}=N\left\langle S_{z}\right\rangle_{T} / S$, where $\left\langle S_{z}\right\rangle_{T} / S=\tanh \hbar \Omega_{S} / k_{\mathrm{B}} T$ is the spin polarization. The solid lines are least-squares fits based on eq 2 that extrapolate to $G_{N} / 2 \pi \simeq 2.3$ $\mathrm{MHz}$ for $T \rightarrow 0$ (when $N_{\text {eff }} \rightarrow N$ ).

$65 \mathrm{MHz}$ is 5 times larger than what would be expected from the spin coherence times. The additional broadening probably arises from the smaller size of the DPPH nanoaggregates 
transferred to this device and from the fact that DPPH molecules are here dispersed in a matrix of glycerol used in the DPN deposition process (see the section Methods below and the Supporting Information (SI) for more details). As mentioned above, these effects tend to suppress direct exchange interactions between free-radical spins and, then, enhance the broadening associated with hyperfine and dipoledipole couplings.

As expected, the spin-photon coupling strength $G_{N}$ decreases rapidly with increasing temperature. This dependence can be accounted for by the decrease in spin polarization that determines $N_{\text {eff }} / N$ (cf. Figure 5D and 5E), showing again the validity of eq 2 . From these data, and using the topographic and geometrical information on the sample, we estimate an average single spin coupling $G_{1} / 2 \pi \simeq 180 \mathrm{~Hz}$. This represents an enhancement of nearly 2 orders of magnitude with respect to the coupling achieved for a $14 \mu \mathrm{m}$ wide transmission line resonator.

Taking into account that the photon energy, thus also $G_{1} \propto$ $\omega_{\mathrm{r}}$, it is useful to define the dimensionless coupling $G_{1} / \omega_{\mathrm{r}} \simeq$ $1.3 \times 10^{-7}$. This result compares favorably to the maximum single-spin coupling reported in the literature, of about $450 \mathrm{~Hz}$ for a $7 \mathrm{GHz}$ resonator (for a ratio $G_{1} / \omega_{\mathrm{r}} \simeq 6.4 \times 10^{-8}$ ), ${ }^{5}$ which was achieved with especially designed lumped-element resonators coupled to impurity spins in the $\mathrm{Si}$ substrate on which these devices were fabricated. Yet, it still falls short of the maximum theoretically attainable enhancement, which should scale as $1 / w$ and therefore reach a factor of 330 in this case, thus $G_{1} / 2 \pi$ close to $1 \mathrm{kHz} .^{16,20}$

In order better understand these results and, in particular, obtain information on how each molecular spin couples to the resonator, we have performed numerical simulations of the spin-photon interaction for this specific situation. The model uses the actual geometry (length, thickness, and width) of the nanoconstriction and a three-dimensional map of the number of spins extracted from the combined analysis of SEM and AFM images. The region surrounding the constriction is divided into a grid of cubic cells with lateral dimensions $d$. Further details are given in the section Methods and as part of the Supporting Information.

A $2 \mathrm{D}$ projection of a map calculated for the smallest $d=3$ $\mathrm{nm}$ is shown in Figure 4C. The coupling is then evaluated as follows. First, the photon energy $\hbar \omega_{\mathrm{r}}$ is used to determine the supercurrent flowing through the constriction. This electrical current generates a magnetic field $\mu_{0} \vec{h}(\vec{r})$ at each point in space $\vec{r}$, which was calculated using the 3D-MLSI finiteelement computer simulation software ${ }^{35}$ as in the example shown in Figure 1B. For all molecules in a given cell $i$, the magnetic field is assigned its value $\mu_{0} \vec{h}_{i}$ at the center of the cell. Then, the coupling of each cell is calculated with the following expression (for details, see ref 20)

$$
G_{i}=g \mu_{\mathrm{B}} \sqrt{n_{i}}\left|\left\langle m_{S}=+1 / 2\left|\mu_{0} \overrightarrow{h_{i}} \cdot \vec{S}\right| m_{S}=-1 / 2\right\rangle\right|
$$

where $n_{i}$ is the effective number of spins in the cell, calculated at the given temperature, and $m_{S}= \pm 1 / 2$ denote the two eigenstates associated with opposite projections of the radical spins along the external magnetic field $\vec{H}$. The contributions of all cells can then be combined to estimate the collective coupling of the whole sample as follows ${ }^{47}$

$$
G_{N}=\sqrt{\sum_{i} G_{i}^{2}}
$$

For the device shown in Figure 4, eq 4 gives $G_{N} / 2 \pi \simeq 2.0$ $\mathrm{MHz}$ at $44 \mathrm{mK}$ and an average $G_{1} \simeq 200 \mathrm{~Hz}$, which agree very well with the experimental values $G_{N}=1.9 \mathrm{MHz}$ and $G_{1}=180$ $\mathrm{Hz}$ and, as shown in Figure 5B, account well for the transmission experiments. The difference is in fact smaller than the unavoidable errors associated with the number of molecules and with the influence that crystalline defects have on the free radicals, which are known to turn a fraction of molecules into a diamagnetic state.

Simulations can then help in understanding how these average values ensue from the distribution of spin locations and couplings in the deposit. A projection map showing the maximum coupling obtained for cells located at different $x y$ positions (which mainly correspond to those lying closest to the device surface along the vertical axis $z$ ) is shown in Figure $4 D$. It confirms that the main contribution to $G_{N}$ comes from those spins located in the closest proximity to the constriction. It also shows that individual couplings significantly larger than the average $G_{1}$ can be achieved. Looking at those spins lying closer to the constriction, we find values as high as $0.6-0.8$ $\mathrm{kHz}$, depending on the exact location with respect to the surface and on the orientation of the external magnetic field.

Spin-Photon Coupling versus Transmission Line Width. Results obtained for different devices enable determining how the single spin to single photon coupling $G_{1}$ depends on the dimensions of the superconducting transmission line. This dependence is shown in Figure 6,

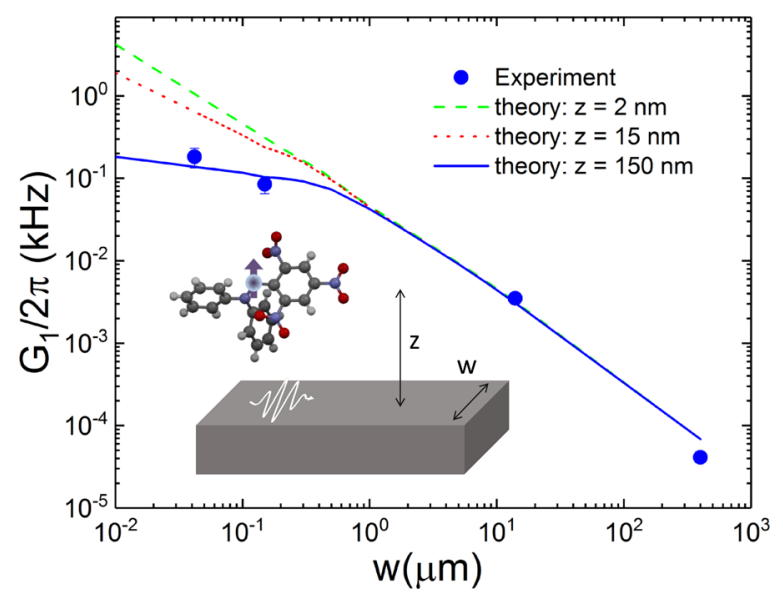

Figure 6. Dependence of the average single spin to single photon coupling on the width of the central transmission line of the resonator, showing the enhancement obtained by reducing the latter down to the region of (tens of) nanometers. The lines are calculations of the coupling of a spin located over the constriction, at three different heights $z$, as illustrated by the figure in the inset.

which, besides those discussed already, includes also data measured on a resonator with a $400 \mu \mathrm{m}$ wide line (cf. Supporting Information). For $w \geqslant 100 \mathrm{~nm}$, these results show the expected enhancement of $G_{1} \propto 1 / w$, from $G_{1} \leqslant 5 \mathrm{mHz}$ up to nearly $100 \mathrm{~Hz}$. For narrower lines, however, and as we have already pointed out above, the increase slows down.

The reason for this can be understood in light of the simulations and arguments described in the previous subsection (cf. Figure 4D). A first limitation arises from the 
nonzero thickness of the superconducting film (nominally 150 $\mathrm{nm}$ but decreasing near the constriction as shown in Figure S2). This geometrical effect reduces slightly the local magnetic field generated by the supercurrents and, as a result, also the slope of the $G_{1}$ vs $1 / w$ theoretical curve of Figure 6 when $w$ becomes smaller than the $\mathrm{Nb}$ thickness. It is, however, unable to account for the experimental results (see Figure S16 in the SI).

Since the coupling enhancement is concentrated close to the constriction, within a region with dimensions comparable to $w$, the sample integration becomes critical. The topography AFM images show that, once dry, DPPH molecules deposited by DPN tend to form nanoaggregates with characteristic dimensions of the order of $200 \mathrm{~nm}$. Then, even those molecules located in grains that land next to the line can be too far when compared to $w$. This effect can be accounted for by numerical calculations of $G_{1}$ (Figures 6 and S16). They show that $G_{1}$ strongly depends on the height $z$ of the molecular spins above the transmission line. While, for $z \simeq 0, G_{1} \propto 1 / w$, when $z \neq 0$ the coupling tends to saturate as soon as $w \leqslant z$. Therefore, creating an optimum interface between the molecular deposits and the circuit surface, i.e. making $z$ as close to zero as possible, becomes of utmost importance to attain the maximum spin-photon coupling.

\section{CONCLUSIONS}

The experiments and simulations described in the previous sections validate a relatively straightforward method to enhance the coupling of molecular spins to on-chip superconducting resonators. Using conventional $1.4 \mathrm{GHz}$ coplanar resonators, we have attained an average single spin coupling of about $180 \mathrm{~Hz}$ for central line widths of $\sim 40 \mathrm{~nm}$, with estimated maximum couplings on the order of $\mathrm{kHz}$. This approach is, in principle, applicable to any circuit design and to a large variety of samples, provided that they can be delivered from solution and with sufficient spatial accuracy. Introducing superconducting nanobridges into especially designed resonators, which minimize the circuit impedance and therefore maximize the superconducting current at the inductor, ${ }^{4-6}$ should allow enhancing $G_{1}$ by a further 2 orders of magnitude, thus reaching $G_{1} / 2 \pi$ values close to $0.1 \mathrm{MHz}$.

Devices based on these ideas can take electron spin spectroscopy to the single spin limit and become useful tools to investigate magnetic excitations in individual nanosystems ranging from nanosized particles to molecular nanomagnets, or even exotic topological states, such as vortices and skyrmions. ${ }^{48}$ The molecular approach used here adds the possibility of serving as a suitable vehicle to deliver diverse samples, as well as to improve their interface with the circuit. A recent work shows that it is possible to synthesize thin molecular films onto a superconducting line, thereby achieving a close to optimum coverage with a minimum molecule to circuit distance. $^{28}$ The combination of these methods with DPN or other nanolithography tools with a high spatial resolution could then lead to major improvements.

Spins hosted in artificial molecules ${ }^{29-34,49}$ and 2D molecular materials, such as graphene nanoribbons, ${ }^{50,51}$ are also very promising candidates to encode qubits and qudits. In recent times, spin coherence times of these systems have been optimized by chemical design, up to values well above 10-50 $\mu \mathrm{s},{ }^{52-55}$ and even close to $\mathrm{ms},{ }^{56}$ and the strong coupling to superconducting resonators has been achieved for macroscopic molecular ensembles. ${ }^{57-59}$ Our results show that attaining this regime for single molecules is within reach. This coherent coupling would allow the use of superconducting circuits to control, read-out, and connect spin qubits located in different molecules, an essential ingredient of a hybrid architecture for large scale quantum computation and simulation. ${ }^{16}$

\section{METHODS}

Device Fabrication and Characterization. Superconducting resonators are fabricated on $500 \mu \mathrm{m}$ thick C-plane sapphire wafers. A $150 \mathrm{~nm}$ thick niobium layer was deposited by radio frequency sputtering and then patterned by photolithography and reactive ion etching. The circuits consist of a large $400 \mu \mathrm{m}$ central line separated from two ground planes by two $200 \mu \mathrm{m}$ gaps that narrow down to around 14 and $7 \mu \mathrm{m}$, respectively, after going through gap capacitors. The devices were tuned to show an effective impedance $Z_{0}=50 \Omega$, a resonance frequency $\omega_{\mathrm{r}} / 2 \pi \simeq 1.4 \mathrm{GHz}$, and maximum quality factors $Q \simeq(1-2) \times 10^{5}$.

Nanoscale constrictions ${ }^{21}$ were made at the midpoint of the central line by etching it with a focused beam of $\mathrm{Ga}^{+}$ions, using a commercial dual beam system. The ion beam also locally reduces the $\mathrm{Nb}$ thickness below the initial $150 \mathrm{~nm}$, even down to values close to the constriction width $w$ (see Figure S2 in the Supporting Information for an illustrative image of the smallest constriction used in this work). The ionic current was kept below $20 \mathrm{pA}$ to maximize the resolution in the fabrication process and to minimize the $\mathrm{Nb}$ layer, on the order 10 to $15 \mathrm{~nm}$ thick, that is implanted with Ga. In order to avoid the buildup, and eventual discharge, of electrostatic charges during the process, the central line was connected to one of the ground planes by a few nanometer wide $\mathrm{Pt}$ bridge, fabricated by focused ion beam deposition. Once the nanoconstriction was fabricated, this bridge was removed using the same ion beam. Images of 158 and $42 \mathrm{~nm}$ wide constrictions, obtained in situ by SEM, are shown in Figure 1 and in the Supporting Information.

Prior to the deposition of the molecular samples, the microwave propagation trough all devices was characterized, as described below, as a function of magnetic field, temperature, and input power. Illustrative results are shown as part of the Supporting Information. These experiments show that the constrictions do not introduce any drastic changes to $\omega_{\mathrm{r}}$ and $Q$ and that they can work under magnetic fields up to $1.4 \mathrm{~T}$, provided that they are applied parallel to the plane of the chip.

Molecular Pattering onto Superconducting Resonators and Characterization of the Deposits. The molecular ink used in all depositions was prepared by dissolving $10-20 \mathrm{mg} / \mathrm{mL}$ of the free radical 2,2-diphenyl-1-picrylhydrazyl (DPPH; Sigma-Aldrich) in $N, N$ Dimethylformamide (DMF; Sigma-Aldrich) with about 5-10\% glycerol in volume (Panreac, $\geq 99.5 \%$, ACS grade). DMF preserves the chemical properties of DPPH and does not turn the radical into its diamagnetic form. Glycerol is used as an additive to increase the viscosity and slow down the evaporation of the ink so that the solution does not dry before the creation of the pattern. ${ }^{42}$ This is especially important in the deposition by DPN, when the drop has to be transferred to the device by the AFM tip.

The device was previously cleaned with isopropanol and acetone, dried by blowing nitrogen gas, and underwent plasma oxygen treatment before deposition. DPN deposition was performed using a DPN5000 based-AFM system (NanoInk, Inc., USA). For this, $1 \mu \mathrm{L}$ of the ink solution was left in a reservoir of a microfluidic Inkwell delivery chip-based system (Acs-technologies LLC, USA). Afterward, a DPN silicon nitride DPN single A-S2 probe (Acs-technologies LLC, USA) was dipped and coated several times with the ink at the inkwell microchannel.

DPN patterning was optimized at $25{ }^{\circ} \mathrm{C}$ and $55 \%$ relative humidity. The quality and size of the free radical deposits were assayed previously on marked $\mathrm{SiO}_{2}$ substrates and small pieces of muscovite mica (see Supporting Information for illustrative images of the results). The deposition conditions were optimized to produce deposits ranging from 1 to $60 \mu \mathrm{m}$ in diameter, such as the one shown in Figure S8. Then, different drops with diameters ranging between 5 
and $60 \mu \mathrm{m}$ and heights from 50 to $400 \mathrm{~nm}$ were patterned on the central line of resonators, both without constriction and with nanoconstrictions. The characterization of the deposits and the microwave transmission experiments were performed once the deposits had dried. Free radical deposits were also made far from the sensing areas and using different solvents and additives and characterized as controls.

The patterns were analyzed by optical microscopy, AFM and SEM imaging. AFM images were taken with a MultiMode 8 AFM system (Bruker) and a Cervantes SPM FullMode (Nanotec) at scan rates of 0.1-2.0 Hz. The samples were characterized using Peak Force TappingTM and TappingTM modes with $7-125 \mathrm{kHz}$ triangular silicon nitride microlevers (SNL; Bruker Probes) having ultrasharp 2 $\mathrm{nm}$ end tip radii, and with an antimony (n) doped silicon $320 \mathrm{kHz}$ ultraresonant microlever (TESP; Bruker Probes) having an $8 \mathrm{~nm}$ end tip radii. The images were analyzed using the $\mathrm{WSxM}^{60}$ and Gwyddion $^{61}$ software for SPM image processing. SEM images were performed with an INSPECT-F50 (FEI).

Microwave Transmission Experiments. The microwave transmission measurements were done using a programmable network analyzer. Experiments performed at $4.2 \mathrm{~K}$ were aimed at measuring a large number of deposits of varying dose. In these experiments, the devices were mounted on a homemade probe and submerged in a liquid helium cryostat. The input power was attenuated by $-52 \mathrm{~dB}$, to avoid the possibility of exceeding the critical current at the nanoconstriction, and the output power was measured directly. The number of photons in the cavity was estimated to be on the order of $10^{9}$. The external magnetic field was applied with a commercial $9 \mathrm{~T} \times$ $1 \mathrm{~T} \times 1 \mathrm{~T}$ superconducting vector magnet, which allows rotating $\vec{H}$ in situ, in order to align it along the central line of the device ( $y$ axis in Figures 1 and 4) with an accuracy better than $0.1^{\circ}$.

For experiments at lower temperatures, transmission measurements were carried out in an adiabatic demagnetization refrigerator, having a base temperature of about $45 \mathrm{mK}$. Magnetic fields up to $60 \mathrm{mT}$ were applied using a homemade superconducting vector magnet. In this case, experimental constraints imposed a field orientation along the $x$ axis, thus perpendicular to the central line within the plane of the device. As it is shown in the Supporting Information, this geometry leads to a substantial decrease in the spin-photon coupling with respect to the maximum achievable. The input signal was attenuated, down to a level of about $5 \times 10^{5}$ photons, and then amplified at $4.2 \mathrm{~K}$ and at room temperature before being detected.

The small asymmetry in the resonances measured (Figures 3 and 5 ) is likely caused by an interference, called Fano resonance, ${ }^{62}$ between the resonator and a small continuous background. A possible source for that background is the off-resonant tail of the sample box mode. This small asymmetry has a very weak influence (of a few \% at most) on the results for the coupling and peak width, because they are first-order independent.

Numerical Simulations. Our estimate of the spin-photon coupling relies on the calculation of the spatial distribution of $\mu_{0} \vec{h}(\vec{r})$, i.e., the rms magnetic field generated by the rms supercurrent $i_{\mathrm{sc}}=\omega_{\mathrm{r}} \sqrt{\pi \hbar / 4 Z_{0}}=11.3 \mathrm{nA}$ circulating through the central line of the resonator, with $Z_{0} \approx 50 \Omega$ its impedance. ${ }^{20}$

The spatial distribution of $i_{\mathrm{sc}}(\vec{r})$ is computed using a finite-element based package ${ }^{35}$ that solves the London equations for the given geometry of the superconducting wire, having London penetration depth $\lambda_{\mathrm{L}}=90 \mathrm{~nm}$, and assuming that the supercurrent distributes through 11 equidistant $2 \mathrm{D}$ sheets, parallel to the $x y$ plane and homogeneously distributed across the thickness of the Nb layer. $i_{\mathrm{sc}}(\vec{r})$ is then used to evaluate the rms magnetic field $\mu_{0} \vec{h}_{i}$ (with $i=1,2, \ldots$ $N_{\text {cell }}$ ) at the center of $N_{\text {cell }}$ cubic cells with lateral dimension $d$, each containing $n_{i}$ molecules. The grid size was varied from 0 up to $2 \mu \mathrm{m}$, and the cell size $d$, from $3 \mathrm{~nm}$ up to $100 \mathrm{~nm}$. The constriction geometry, in particular its thickness, was found to have some influence on the coupling, but only for spins located very close to the constriction and for $w \leq 50 \mathrm{~nm}$.
These simulations show also that spins located further than about $500 \mathrm{~nm}$ from the constriction give a close to negligible contribution. The mode volume of a $42 \mathrm{~nm}$ wide constriction is then $\simeq 1 \mu \mathrm{m} \times 1$ $\mu \mathrm{m} \times 10 \mu \mathrm{m}=10 \mu \mathrm{m}^{3}=0.01$ pico-1, or about $1.4 \times 10^{-14} \lambda^{3}$, with $\lambda \simeq$ $88 \mathrm{~mm}$ being the resonance wavelength. It also follows that a $3 \mathrm{~nm}$ cell size is necessary to properly account for the coupling to spins that lie very close to the nanoconstriction, which give the maximum contribution. The maximum coupling $G_{1}$ depends also on the orientation of the external magnetic field, which determines which components of the photon magnetic field couple to the spins. It is maximum for $\vec{H}$ parallel to the central line ( $y$ axis). Further details can be found in the Supporting Information (Figures S12 to S16).

\section{ASSOCIATED CONTENT}

\section{Supporting Information}

The Supporting Information is available free of charge at https://pubs.acs.org/doi/10.1021/acsnano.0c03167.

Images of the fabrication steps of resonators and of superconducting nanoconstrictions, tests results of these devices, details and additional images of the deposition of free-radical spin ensembles by DPN and their characterization by means of SEM and AFM, results of additional microwave transmission experiments, additional information, backed with plots, on how the number of molecules effectively coupled to each device has been estimated and how the spin-photon coupling has been simulated (PDF)

\section{AUTHOR INFORMATION}

\section{Corresponding Author}

Fernando Luis - Instituto de Ciencia de Materiales de Aragón, CSIC-Universidad de Zaragoza, 50009 Zaragoza, Spain; ○ orcid.org/0000-0001-6284-0521; Email: fluis@unizar.es

\section{Authors}

Ignacio Gimeno - Instituto de Ciencia de Materiales de Aragón, CSIC-Universidad de Zaragoza, 50009 Zaragoza, Spain

Wenzel Kersten - Vienna Center for Quantum Science and Technology, Atominstitut, TU Wien, 1020 Vienna, Austria

María C. Pallarés - Laboratorio de Microscopías Avanzadas, Instituto de Nanociencia de Aragón, Universidad de Zaragoza, 50018 Zaragoza, Spain

Pablo Hermosilla - Laboratorio de Microscopías Avanzadas, Instituto de Nanociencia de Aragón, Universidad de Zaragoza, 50018 Zaragoza, Spain

María José Martínez-Pérez - Instituto de Ciencia de Materiales de Aragón, CSIC-Universidad de Zaragoza, 50009 Zaragoza, Spain; Fundación ARAID, 50018 Zaragoza, Spain; (i) orcid.org/0000-0002-8125-877X

Mark D. Jenkins - Instituto de Ciencia de Materiales de Aragón, CSIC-Universidad de Zaragoza, 50009 Zaragoza, Spain

Andreas Angerer - Vienna Center for Quantum Science and Technology, Atominstitut, TU Wien, 1020 Vienna, Austria

Carlos Sánchez-Azqueta - Departamento de Física Aplicada, Universidad de Zaragoza, 50009 Zaragoza, Spain

David Zueco - Instituto de Ciencia de Materiales de Aragón, CSIC-Universidad de Zaragoza, 50009 Zaragoza, Spain; Fundación ARAID, 50018 Zaragoza, Spain

Johannes Majer - Shanghai Branch, CAS Center for Excellence and Synergetic Innovation Center in Quantum Information and Quantum Physics and National Laboratory for Physical Sciences at Microscale and Department of Modern Physics, University of Science and Technology of China, Shanghai 
201315, China; Vienna Center for Quantum Science and Technology, Atominstitut, TU Wien, 1020 Vienna, Austria

Anabel Lostao - Instituto de Ciencia de Materiales de Aragón, CSIC-Universidad de Zaragoza, 50009 Zaragoza, Spain; Laboratorio de Microscopías Avanzadas, Instituto de Nanociencia de Aragón, Universidad de Zaragoza, 50018 Zaragoza, Spain; Fundación ARAID, 50018 Zaragoza, Spain; (1) orcid.org/0000-0001-7460-5916

Complete contact information is available at: https://pubs.acs.org/10.1021/acsnano.0c03167

\section{Author Contributions}

The experiments were conceived and designed by J.M., A.L. and F.L. The superconducting devices were designed and fabricated by I.G., M.J., J.M., and F.L. The molecular deposition and subsequent AFM and SEM characterization were carried out by M.C.P., P.H., and A.L. The microwave transmission experiments were carried out by I.G., W.K., A.A., C.S.-A., M.J., J.M., and F.L. Simulations of the spin-photon coupling were carried out by M.J.M.-P. and by D.Z. F.L. wrote the manuscript with input from all coauthors. I.G., W.K., and M.C.P. contributed equally to this work.

\section{Notes}

The authors declare no competing financial interest.

\section{ACKNOWLEDGMENTS}

The authors acknowledge funding from the EU (COST Action 15128 MOLSPIN, QUANTERA SUMO and MICROSENSE projects, FET-OPEN Grant 862893 FATMOLS), the Spanish MICINN (Grants RTI2018-096075-B-C21, PCI2018-093116, MAT2017-89993-R, MAT2017-88358-C3-1-R, EUR2019103823), the Gobierno de Aragón Ggrants E09-17R QMAD, E35-20R, BE and LMP55-18, FANDEPAM) and the BBVA foundation (Leonardo Grants 2018 and 2019).

\section{REFERENCES}

(1) Wallraff, A.; Schuster, D. I.; Blais, A.; Frunzio, L.; Huang, R.-S.; Majer, J.; Kumar, S.; Girvin, S. M.; Schoelkopf, R. J. Strong Coupling of a Single Photon to a Superconducting Qubit Using Circuit Quantum Electrodynamics. Nature 2004, 431, 162-167.

(2) Göppl, M.; Fragner, A.; Baur, M.; Bianchetti, R.; Filipp, S.; Fink, J. M.; Leek, P. J.; Puebla, G.; Steffen, L.; Wallraff, A. Coplanar Waveguide Resonators for Circuit Quantum Electrodynamics. J. Appl. Phys. 2008, 104, 113904.

(3) Bienfait, A.; Pla, A. A.; Kubo, Y.; Stern, M.; Zhou, X.; Lo, C. C.; Weis, C. D.; Schenkel, T.; Thewalt, M. L. W.; Vion, D.; Esteve, D.; Julsgaard, B.; Mølmer, K.; Morton, J. J. L.; Bertet, P. Reaching the Quantum Limit of Sensitivity in Electron Spin Resonance. Nat. Nanotechnol. 2016, 11, 253-257.

(4) Eichler, C.; Sigillito, A. J.; Lyon, S. A.; Petta, J. R. Electron Spin Resonance at the Level of $10^{4}$ Spins Using Low Impedance Superconducting Resonators. Phys. Rev. Lett. 2017, 118, 037701.

(5) Probst, S.; Bienfait, A.; Campagne-Ibarcq, P.; Pla, J. J.; Albanese, B.; Da Silva Barbosa, J. F.; Schenkel, T.; Vion, D.; Esteve, D.; Mølmer, K.; Morton, J. J. L.; Heeres, R.; Bertet, P. Inductive-Detection Electron-Spin Resonance Spectroscopy with 65 Spins/Hz Sensitivity. Appl. Phys. Lett. 2017, 111, 202604.

(6) Sarabi, B.; Huang, P.; Zimmerman, N. M. Possible Hundredfold Enhancement in the Direct Magnetic Coupling of a Single-Atom Electron Spin to a Circuit Resonator. Phys. Rev. Appl. 2019, 11, 014001 .

(7) Blais, A.; Huang, R.-S.; Wallraff, A.; Girvin, S. M.; Schoelkopf, R. J. Cavity Quantum Electrodynamics for Superconducting Electrical Circuits: An Architecture for Quantum Computation. Phys. Rev. A: At., Mol., Opt. Phys. 2004, 69, 062320.
(8) Majer, J.; Chow, J. M.; Gambetta, J. M.; Koch, J.; Johnson, B. R.; Schreier, J. A.; Frunzio, L.; Schuster, D. I.; Houck, A. A.; Wallraff, A.; Blais, A.; Devoret, M. H.; Girvin, S. M.; Schoelkopf, R. J. Coupling Superconducting Qubits via a Cavity Bus. Nature 2007, 449, 443447.

(9) Schoelkopf, R. J.; Girvin, S. M. Wiring Up Quantum Systems. Nature 2008, 451, 664-669.

(10) Imamoğlu, A. Cavity QED Based on Collective Magnetic Dipole Coupling: Spin Ensembles as Hybrid Two-Level Systems. Phys. Rev. Lett. 2009, 102, 083602.

(11) Wesenberg, J. H.; Ardavan, A.; Briggs, G. A. D.; Morton, J. J. L.; Schoelkopf, R. J.; Schuster, D. I.; Mølmer, K. Quantum Computing with an Electron Spin Ensemble. Phys. Rev. Lett. 2009, 103, 070502.

(12) Schuster, D. I.; Sears, A. P.; Ginossar, E.; DiCarlo, L.; Frunzio, L.; Morton, J. J. L.; Wu, H.; Briggs, G. A. D.; Buckley, B. B.; Awschalom, D. D.; Schoelkopf, R. J. High-Cooperativity Coupling of Electron-Spin Ensembles to Superconducting Cavities. Phys. Rev. Lett. 2010, 105, 140501.

(13) Kubo, Y.; Ong, F. R.; Bertet, P.; Vion, D.; Jacques, V.; Zheng, D.; Dréau, A.; Roch, J.-F.; Auffeves, A.; Jelezko, F.; Wrachtrup, J.; Barthe, M. F.; Bergonzo, P.; Esteve, D. Strong Coupling of a Spin Ensemble to a Superconducting Resonator. Phys. Rev. Lett. 2010, 105, 140502.

(14) Wu, H.; George, R. E.; Wesenberg, J. H.; Mølmer, K.; Schuster, D. I.; Schoelkopf, R. J.; Itoh, K. M.; Ardavan, A.; Morton, J. J. L.; Briggs, G. A. D. Storage of Multiple Coherent Microwave Excitations in an Electron Spin Ensemble. Phys. Rev. Lett. 2010, 105, 140503.

(15) Chiorescu, I.; Groll, N.; Bertaina, S.; Mori, T.; Miyashita, S. Magnetic Strong Coupling in a Spin-Photon System and Transition to Classical Regime. Phys. Rev. B: Condens. Matter Mater. Phys. 2010, 82, 024413.

(16) Jenkins, M. D.; Zueco, D.; Roubeau, O.; Aromí, G.; Majer, J.; Luis, F. A Scalable Architecture for Quantum Computation with Molecular Nanomagnets. Dalton Trans 2016, 45, 16682-16693.

(17) Narkowicz, R.; Suter, D.; Stonies, R. Planar Microresonators for EPR Experiments. J. Magn. Reson. 2005, 175, 275-284.

(18) Narkowicz, R.; Suter, D.; Niemeyer, I. Scaling of Sensitivity and Efficiency in Planar Microresonators for Electron Spin Resonance. Rev. Sci. Instrum. 2008, 79, 084702.

(19) Banholzer, A.; Narkowicz, R.; Hassel, C.; Meckenstock, R.; Stienen, S.; Posth, O.; Suter, D.; Farle, M.; Lindner, J. Visualization of Spin Dynamics in Single Nanosized Magnetic Elements. Nanotechnology 2011, 22, 295713.

(20) Jenkins, M. D.; Hümmer, T.; Martínez-Pérez, M. J.; GarcíaRipoll, J.; Zueco, D.; Luis, F. Coupling Single-Molecule Magnets to Quantum Circuits. New J. Phys. 2013, 15, 095007.

(21) Jenkins, M. D.; Naether, U.; Ciria, M.; Sesé, J.; Atkinson, J.; Sánchez-Azqueta, C.; del Barco, E.; Majer, J.; Zueco, D.; Luis, F. Nanoscale Constrictions in Superconducting Coplanar Waveguide Resonators. Appl. Phys. Lett. 2014, 105, 162601.

(22) Haikka, P.; Kubo, Y.; Bienfait, A.; Bertet, P.; Mølmer, K. Proposal for Detecting a Single Electron Spin in a Microwave Resonator. Phys. Rev. A: At., Mol., Opt. Phys. 2017, 95, 022306.

(23) Mannini, M.; Pineider, F.; Sainctavit, P.; Danieli, C.; Otero, E.; Sciancalepore, C.; Talarico, A. M.; Arrio, M.-A.; Cornia, A.; Gatteschi, D.; Sessoli, R. Magnetic Memory of a Single-Molecule Quantum Magnet Wired to a Gold Surface. Nat. Mater. 2009, 8, 194-197.

(24) Mannini, M.; Pineider, F.; Danieli, C.; Totti, F.; Sorace, L.; Sainctavit, P.; Arrio, M.-A.; Otero, E.; Joly, L.; Cezar, J. C.; Cornia, A.; Sessoli, R. Quantum Tunnelling of the Magnetization in a Monolayer of Oriented Single-Molecule Magnets. Nature 2010, 468, 417-421.

(25) Domingo, N.; Bellido, E.; Ruiz-Molina, D. Advances on Structuring, Integration and Magnetic Characterization of Molecular Nanomagnets on Surfaces and Devices. Chem. Soc. Rev. 2012, 41, 258-302.

(26) Thiele, S.; Balestro, F.; Ballou, R.; Klyatskaya, S.; Ruben, M.; Wernsdorfer, W. Electrically Driven Nuclear Spin Resonance in Single-Molecule Magnets. Science 2014, 344, 1135-1138. 
(27) Malavolti, L.; Briganti, M.; Hänze, M.; Serrano, G.; Cimatti, I.; McMurtrie, G.; Otero, E.; Ohresser, P.; Totti, F.; Mannini, M.; Sessoli, R.; Loth, S. Tunable Spin-Superconductor Coupling of Spin 1/2 Vanadyl Phthalocyanine Molecules. Nano Lett. 2018, 18, 7955.

(28) Urtizberea, A.; Natividad, E.; Alonso, P. J.; Pérez-Martínez, L.; Andrés, M. A.; Gascón, I.; Gimeno, I.; Luis, F.; Roubeau, O. Vanadyl Spin Qubit 2D Arrays and their Integration on Superconducting Resonators. Mater. Horiz. 2020, 7, 885-897.

(29) Leuenberger, M.; Loss, D. Quantum Computing in Molecular Magnets. Nature 2001, 410, 789-793.

(30) Troiani, F.; Affronte, M. Molecular Spins for Quantum Information Technologies. Chem. Soc. Rev. 2011, 40, 3119-3129.

(31) Aromí, G.; Aguilà, D.; Gamez, P.; Luis, F.; Roubeau, O. Design of Magnetic Coordination Complexes for Quantum Computing. Chem. Soc. Rev. 2012, 41, 537-546.

(32) Moreno-Pineda, E.; Godfrin, C.; Balestro, F.; Wernsdorfer, W.; Ruben, M. Molecular Spin Qudits for Quantum Algorithms. Chem. Soc. Rev. 2018, 47, 501-513.

(33) Gaita-Ariño, A.; Luis, F.; Hill, S.; Coronado, E. Molecular Spins for Quantum Computation. Nat. Chem. 2019, 11, 301-309.

(34) Atzori, M.; Sessoli, R. The Second Quantum Revolution: Role and Challenges of Molecular Chemistry. J. Am. Chem. Soc. 2019, 141, 11339-11352.

(35) Khapaev, M. M.; Kupriyanov, M. Y.; Goldobin, E.; Siegel, M. Current Distribution Simulation for Superconducting Multi-Layered Structures. Supercond. Sci. Technol. 2003, 16, 24-27.

(36) Weil, J. A.; Anderson, J. K. 1039. The Determination and Reaction of 2,2-Diphenyl-1-Picrylhydrazyl with Thiosalicylic Acid. J. Chem. Soc. 1965, 5567-5570.

(37) Yordanov, N. D. Is Our Knowledge about the Chemical and Physical Properties of DPPH Enough to Consider it as a Primary Standard for Quantitative EPR Spectrometry? Appl. Magn. Reson. 1996, 10, 339-350.

(38) Žilić, D.; Pajić, D.; Jurić, M.; Molčanov, K.; Rakvin, B.; Planinić, P.; Zadro, K. Single Crystals of DPPH Grown from Diethyl Ether and Carbon Disulfide Solutions - Crystal Structures, IR, EPR and Magnetization Studies. J. Magn. Reson. 2010, 207, 34-41.

(39) Anderson, P. W.; Weiss, P. R. Exchange Narrowing in Paramagnetic Resonance. Rev. Mod. Phys. 1953, 25, 269-276.

(40) Höcherl, G.; Wolf, H. C. Zur Konzentrationsabhängigkeit der Elektronenspin-Relaxationszeiten von Diphenyl-Picryl-Hydrazyl in fester Phase. Eur. Phys. J. A 1965, 183, 341-351.

(41) Piner, R. D.; Zhu, J.; Xu, F.; Hong, S.; Mirkin, C. A. "Dip-Pen" Nanolithography. Science 1999, 283, 661-663.

(42) Bellido, E.; de Miguel, R.; Ruiz-Molina, D.; Lostao, A.; Maspoch, D. Controlling the Number of Proteins with Dip-Pen Nanolitography. Adv. Mater. 2010, 22, 352-355.

(43) Martínez-Pérez, M. J.; Bellido, E.; de Miguel, R.; Sesé, J.; Lostao, A.; Gómez-Moreno, C.; Drung, D.; Schurig, T.; Ruiz-Molina, D.; Luis, F. Alternating Current Magnetic Susceptibility of a Molecular Magnet Submonolayer Directly Patterned onto a Micro Superconducting Quantum Interference Device. Appl. Phys. Lett. 2011, 99, 032504

(44) Bellido, E.; González-Monje, P.; Repollés, A.; Jenkins, M.; Sesé, J.; Drung, D.; Schurig, T.; Awaga, K.; Luis, F.; Ruiz-Molina, D. $\mathrm{Mn}_{12}$ Single Molecule Magnets Deposited on $\mu$-SQUID Sensors: the Role of Interphases and Structural Modifications. Nanoscale 2013, 5, $12565-12573$.

(45) Bushev, P.; Feofanov, A. K.; Rotzinger, H.; Protopopov, I.; Cole, J. H.; Wilson, C. M.; Fischer, G.; Lukashenko, A.; Ustinov, A. V. Ultralow-Power Spectroscopy of a Rare-Earth Spin Ensemble Using a Superconducting Resonator. Phys. Rev. B: Condens. Matter Mater. Phys. 2011, 84, 060501.

(46) Tavis, M.; Cummings, F. W. Exact Solution for an N-MoleculeRadiation-Field Hamiltonian. Phys. Rev. 1968, 170, 379-384.

(47) Hümmer, T.; Reuther, G. M.; Hänggi, P.; Zueco, D. Nonequilibrium Phases in Hybrid Arrays with Flux Qubits and Nitrogen-Vacancy Centers. Phys. Rev. A: At., Mol., Opt. Phys. 2012, 85, 052320 .
(48) Martínez-Pérez, M. J.; Zueco, D. Strong Coupling of a Single Photon to a Magnetic Vortex. ACS Photonics 2019, 6, 360-367.

(49) Warner, M.; Din, S.; Tupitsyn, I. S.; Morley, G. W.; Stoneham, A.; Gardener, J. A.; Wu, Z.; Fisher, A. J.; Heutz, S.; Kay, C. W. M.; Aeppli, G. Ptential fot Spin-Based Information Processing in a ThinFilm Molecular Semiconductor. Nature 2013, 503, 504-508.

(50) Slota, M.; Keerthi, A.; Myers, W. K.; Tretyakov, E.; Baumgarten, M.; Ardavan, A.; Sadeghi, H.; Lambert, C. J.; Narita, A.; Müllen, K.; Bogani, L. Magnetic Edge States and Coherent Manipulation of Graphene Nanoribbons. Nature 2018, 557, 691-695.

(51) Lombardi, F.; Lodi, A.; Ma, J.; Liu, J.; Slota, M.; Narita, A.; Myers, W. K.; Müllen, K.; Feng, X.; Bogani, L. Quantum Units from the Topological Engineering of Molecular Graphenoids. Science 2019, $366,1107-1110$.

(52) Ardavan, A.; Rival, O.; Morton, J. J. L.; Blundell, S. J.; Tyryshkin, A. M.; Timco, G. A.; Winpenny, R. E. P. Will SpinRelaxation Times in Molecular Magnets Permit Quantum Information Processing? Phys. Rev. Lett. 2007, 98, 057201.

(53) Wedge, C. J.; Timco, G. A.; Spielberg, E. T.; George, R. E.; Tuna, F.; Rigby, S.; McInnes, E. J. L.; Winpenny, R. E. P.; Blundell, S. J.; Ardavan, A. Chemical Engineering of Molecular Qubits. Phys. Rev. Lett. 2012, 108, 107204.

(54) Bader, K.; Dengler, D.; Lenz, S.; Endeward, B.; Jiang, S.-D.; Neugebauer, P.; van Slageren, J. Room Temperature Quantum Coherence in a Potential Molecular Qubit. Nat. Commun. 2014, 5, 5304.

(55) Shiddiq, M.; Komijani, D.; Duan, Y.; Gaita-Ariño, A.; Coronado, E.; Hill, S. Enhancing Coherence in Molecular Spin Qubits via Atomic Clock Transitions. Nature 2016, 531, 348-351.

(56) Zadrozny, J. M.; Niklas, J.; Poluektov, O. G.; Freedman, D. E. Millisecond Coherence Time in a Tunable Molecular Electronic Spin Qubit. ACS Cent. Sci. 2015, 1, 488-492.

(57) Ghirri, A.; Bonizzoni, C.; Troiani, F.; Buccheri, N.; Beverina, L.; Cassinese, A.; Affronte, M. Coherently Coupling Distinct Spin Ensembles through a High- $T$ superconducting Resonator. Phys. Rev. A: At., Mol., Opt. Phys. 2016, 93, 063855.

(58) Mergenthaler, M.; Liu, J.; Le Roy, J. J.; Ares, N.; Thompson, A. L.; Bogani, L.; Luis, F.; Blundell, S. J.; Lancaster, T.; Ardavan, A.; Briggs, G. A. D.; Leek, P. J.; Laird, E. A. Strong Coupling of Microwave Photons to Antiferromagnetic Fluctuations in an Organic Magnet. Phys. Rev. Lett. 2017, 119, 147701.

(59) Bonizzoni, C.; Ghirri, A.; Atzori, M.; Sorace, L.; Sessoli, R.; Affronte, M. Coherent Coupling between Vanadyl Phthalocyanine Spin Ensemble and Microwave Photons: Towards Integration of Molecular Spin Qubits into Quantum Circuits. Sci. Rep. 2017, 7, 13096.

(60) Horcas, I.; Fernández, R. WSXM: A Software for Scanning Probe Microscopy and a Tool for Nanotechnology. Rev. Sci. Instrum. 2007, 78, 013705 .

(61) Nečas, D.; Klapetek, P. Gwyddion: An Open-Source Software for SPM Data Analysis. Centr. Eur. J. Phys. 2012, 10, 181-188.

(62) Fano, U. Effects of Configuration Interaction on Intensities and phase Shifts. Phys. Rev. 1961, 124, 1866-1878. 